very manifest. I refer especially to gout and rheumatism. Both the nasal and buccal secretions are surcharged with the poisons of those diseases periodically; and there can be no doubt that the inflammatory condition of lingual tonsil seen, and central angina and constriction complained of, so often in these diseases, and which often precede other symptoms or are alone present, are due to the irritation of the lingual tonsil by the perverted secretions. Evidence confirmatory of this view is found in the fact that antidiathetic treatment speedily cures the condition. The lingual tonsil does not show the same predisposition to be affected in syphilis as do the faucial tonsils. I venture to suggest that the syphilitic poison is excreted with the buccal secretions rather than with the nasal; and, as has already been stated, it is with the former that the faucial tonsils are in special correlation. An interesting observation bearing on lingual tonsil affection in scarlet fever was recorded to me by a pathologist who asked an explanation. In a fatal case he had observed ulcerations and erosions localised in the area of this organ. This pointed to the fact that the secretions loaded with germs and inflammatory products had vented their fury here, and seems singularly confirmatory of the importance of the lingual tonsil. Taking lupus of the nose and throat again, lupoid infiltrations and ulcerations have been found localised in the lingual tonsil area (Chiari and Reil), the infection having clearly been carried overhealthy parts to a place where it could enter with the secretions and reproduce the disease. When there is nasal obstruction and substituted mouth breathing, all the impurities of the environing atmosphere enter the mouth, and many of them alighting on the mucous membrane are washed on to the lingual and faucial tonsils. Moreover, work is cast on the linings of the mouth-that of warming and moistening the air-which does not belong to them. Hence drying of the surface and failure of the secretions to wash away the decomposing débris. In the morning the patient complains of a dry mouth and a slight wore throat, due to inflanmation of the lingual and faucial tonsils. This at first passes off during the day, but after some time leads to hypertrophy of the irritated structures. Other frequent and potent causes of lingual tonsil abnormalities are tobacco smoking, chewing, and snuffing, their action being irritant to the lymph follicles throughout the upper respiratory tract. Septic influences, bad teeth, meglected dirty teeth, false teeth not kept clean or not well fitting, must be added to the list of common causes of lymph follicle irritation and hypertrophy throughout the whole area under consideration, and must be attended to Wefore a cure can be expected. Lingual tonsil mischief is specially met with in adults, and not so much in children. The reasons I would suggest are that the latter do not indulge in alcohol, condiments, or very hot or very cold fluids; and also that the irritating secretions which in children are penned up in the nose or directed forwards owing to enlarged pharyngeal tonsil to produce a rhinorshoea, in adults pass on (there being more room in the nasopharynx) to the lingual tonsil.

Concerning the faucial tonsils, the special relation of these to the buccal secretions has been referred to, and also that the factors in the production of disease of the other tonsils affect these likewise. It is unnecessary, therefore, to recapitulate, but it remains for me to remark additional facts. Very often unilateral hypertrophy of a faucial tonsil is seen. How often is it not in relation with a carious tooth on the same side, constantly contaminating the buccal secretions on that side? There can be little doubt that the tonsils are the sites where the poison of scarlatina, measles, and diphtheria usually enter the system, since they are the parts first and most constantly, and often alone, visibly affected; and the lymphatic glands in direct communication with them most markedly, soonest, and most frequently involved. Faulty voice production and excessive use of the voice are very important causes of affections of all the tonsils. Excessive use demands excessive lubrication, and the latter implies excessive absorption on the part of all the lymphoid follicles. Hence the diffusiveness, chronicity, and obstinacy of the changes met with in the throats of clergymen, actors, and Board School teachers. Regarding lacunar or so-called follicular tonsillitis (which, by the way, I have noticed more than once well marked in the crypts of the lingual tonsil), there are two distinet varieties elinically. The first is that in which superficial inflammation and swelling of the mucosa blocks the crypt orifices and causes retention of the shed epithelium and débris.
This form is acute and usually easily curable, and may be brought about by septic or common catarrhal causes. It is painful, and requires sedative and antiphlogistic treatment. This variety often forms the starting-point of a lacunar abscess, or the process may extend deeper and peri-tonsillar abscess or quinsy ensue. The second form is chronic, and depends on a natural or acquired sluggishness with which the desquamation processes of the epithelium lining the tonsillar crypts are performed, and the debris is not normally extruded by a vis a tergo. This is not septic or painful, though there may be slight stiffness and discomfort. It is very chronic in course, and difficult to overcome, yield. ing best, however, to solvent and stimulant local applications. The discrete nodules in the pharyngeal walls partake in the morbid processes going on in the other tonsils, and are similarly affected by the causes acting on them.

It now remains for me to state that it has not been my object in this paper to give details of symptoms and treatment, and merely to indicate the lines upon which nose and throat affections should be treated when, as is generally the case, the various tonsils the points of maximum irrita. tion-show palpable signs of morbid action; and it must be observed that, when the various methods of treatment in use in the past are considered in the light of the above views as to the functions of the tonsils, it will be seen that these views afford a scientific explanation of the success of those empirical methods which have been hitherto the most approved. These lines would be, firstly, the ensurance of physiological rest to the affected tissues by arresting morbid and lessening profuse secretions, and promoting derivative action into other channels. In acute and subacute affections, a blue pill, followed by regular small doses of belladonna, gives excellent results. Secondly, the removal of all causes of irritation and inflammation in the inspired air, whether due to occupation, habits, or conditions of existence, and a similar regulation of the habitual alimentary ingesta. Thirdly, the soothing of any acute inflamnation or pain by ordinary measures, ice, bland fluids, jelly, cocaine, \&c. Fourthly, the attack of any diathetic condition which may be causing perversion of secretionsas gout, rheumatism, syphilis, \&c.,-on general principles. Fifthly, the remoral of any hypertrophied tissue-such as enlarged tonsils, post-nasal growths, hypertrophied lingual tonsil, granules of granular pharynx, \&c. - which may be occluding any physiological channel or causing mechanical irritation of adjacent parts by the numerous approved methods at our disposal. Sixthly, the prevention of the accumulation or stagnation of any of the seeretions of the nose, mouth, or pharynx by cleansing and antiseptic washes.

In conclusion I would say-l. The significance of the varions tonsils is in their palpable relation to the bloodmanufacturing system and to the outpour of copious secretions. The relations of the tonsils to the rest of the organism can be well appreciated by comparing then with the relations of the sewage farm to the town whose refuse it makes use of, and to which it returns its elaborated products. 2. If any of the secretions delivered to the tonsils become contaminated in any way with irritating matters, whether generated in the system or introduced from without, those tonsils in physiological correlation with the affected secretion show irritative changes varying in degree. 3. The functions and affections of the various tonsils afford the key to the comprehension and scientific treatment-and the prevention-of many of the most intractable and recurrent disorders of the nose and throat.

Welbeck-street, Cavendish-square, w.

\section{ON A GROUP OF CASES TREATED WITH STROPHANTHUS HISPIDUS.}

\section{By DAVID G. EVANS, M.D., C.M. Edin.}

THE most accurate method of judging the value of any drug in curing a disease or in relieving symptoms is ascertained by carefully watching the changes it produces in the clinical phenomena of a case. By this form of observation we are able to elucidate theories and to convert them into therapentical facts. A scrupulous study of a few wellselected cases has precedence over the off-handed manner of arriving at a conclusion by a bare statistical majority. The following cases are small in number, but I believe that my 
readers will find them to be correctly adopted, and also studied with sufficient minuteness to illustrate pretty fully the action of strophanthus on the cardiac nuscle.

CAsE 1.-On Sept. 29th, 1887, I was desired to visit A. $\mathrm{N}-$, a military gentleman of great reputation, aged sixty-eight years, who complained of breathlessness, which was greatly aggravated on any exertion. He also com. plained of palpitation, occasional fits of dry coughing, and a feeling of tightness about the upper region of his chest. He was likewise very weak and languid. Hereditary tenilencies pointed to gout. The breathlessness first appeared in August, 1886 . Objective examination exin height, and 11 st. $9 \mathrm{lb}$. in weight. Face pale, with an expression of being depressed; and the mouth open, through which he respired. Cyanosis of the alre nasi, lips, ears, and nails. A few dilated venules were distributed on each cheek, and the veins of his hands were over-distended with blood. The conjunctivæ were somewhat yellow. On inspecting the præecordial region, a systolic undulatory movement was perceptible in the upper part of the epigastrium, extending for about three inches along the lower border of the lowest cartilage. A slight visible impulse was also present between the sixth and seventh ribs four inches from the middle line of the sternum, and running along the sixth interspace for an inch. Palpation conveyed an irregular action of the heart to the hand, with a distinct thrill accompanying each systole. The cardiac impulse was diffused, and to be felt in the fourth, fifth, and sixth intercostal spaces; it was greatly wanting in force. The poist of maximum intensity was in the sixth intercostal space, four inches and a half from the middle line of the trunk, considerably to the left of the nipple line. The absolute and relative cardiac dulness was increased principally downwards and to the left, also to the right of the sternum in a less degree. There was a systolic murmur in the mitral area almost entirely replacing the first heart sound, and which was propagated towards the angle of the left scapula and back; it was very audible over the left auricular appendix. There was also a slightly presystolic rough murmur in the same area. The pulse was 86 , rhythm irregular, and in character it was small, feeble, and very compressible. The respiratory system afforded signs of extreme congestion of both lungs. The urine was diminished in quantity; of a muddy yellow colour; sp. gr. 1025 ; reaction acid; boiling a filtered specimen yielded about half its volume of albumen; the only deposits present were amorphous urates. My diagnosis was mitral incompetence, with failure in compensation, and dilatation of both ventricles, especially the right one. There was great congestion of both lungs, and also to a little less extent of the liver, stomach, kidneys, and to a still less degree of the spleen. The treatment consisted in the administration of the following mixture: Tincture of strophanthus, $2 \frac{1}{2} \mathrm{dr}$.; spirits of chloroform, $2 \mathrm{dr}$.; compound infusion of gentian, $6 \mathrm{oz}$; t two teaspoonfuls to be taken in a little water every three hours. The diet ordered consisted of beef-tea, peptonised milk gruel, eggs beaten up and in custards, milk puddings, fish, \&c. On Sept. 30th, the patient was feeling a shade better. Pulse 82. He had taken a little more food. On Oct. 1st he felt that he was improving. Pulse 80. Medicine repeated. On the 2 nd he was still improving. Pulse 78. On the 3rd he was decidedly better, the pulse being stronger, and there was less dyspnoea and palpitation. The dis agreeable sensation of tightness at the upper part of his chest was subsiding. To cut the history short, the patient was well enough to leave off taking the medicine on Nor. 9th, when he stated that he felt better than he had done for over a year. He remained apparently well until Feb. 7th, when I was sent for again, as he com plained of returning symptoms, and $\mathrm{I}$ prescribed the fol fowing: Tincture of strophanthus, tincture of nux vomica, and spirit of chloroform, of each one drachm and $a$ half compound infusion of rentian to eicht ounces; a tablespoonful to be taken three times a day. The patient, unable to wait until my next visit, wrote to me on the $9 \mathrm{th}$, saying what a great amount of benefit he was deriving from taking this mixture, and hoped very much that I would continue it. However, three bottles of the above succeeded in re-establishing compensation, when he felt well enongh to discontinue it again. The pulse was now perfectly regular in rhythm, and strong. The tongue was clean, and appetite good. The liver was smaller, and acting well. There was no congestion of his lungs, and no albumen in the urine.
The patient has remained well since, without any returning symptoms.

Cist 2.-Mrs. V-, aged forty-nine years, complained of being short of breath and of palpitation, which were greatly increased on exertion; she had also a violent cough and anasarca. The patient stated that she had been ailing more or less for two years and a half. She had an attack of bronchitis about Christmas, 1886, and has also had two subsequent ones. She has suffered from rheumatism at times within the last six years, and had an acute attack thirteen years ago. There is a family history of rheumatism on her father's side. Her dyspnoea commenced about two years and a half aco, and was never properly cured, although treated by several medical gentlemen. Heicht $5 \mathrm{ft}$. 2 in. weight $177 \mathrm{lb}$. Well nourished, with a moderate amount of alliposity. Cyanosis of lips, cheeks, ears, and nails. CEdenia of feet, legs, abdominal walls, and chest. Slight undulatory movements synchronous with the ventricular systole were visible in the upper part of the epigastrium. Swelling and pulsations of both right and left jugular veins were present. The action of the heart was very weak, extremely irregular, and at times also intermittent; systolic thrill in the mitral area; percussion dulness increased chiefly downwards and to the left, and no sense of resistance. On auscultation, there was a mitral systolic murmur completely replacing the first cardiac sound and propagated to the maxilla and back, and it could be distinctly heard in the left vertebral groove. The pulse was extremely irrecular, at times intermittent, and also very weak. She had congestion of both lungs and bronchi, caused by the backward pressure on the circulation; this same pressure told on the portal circulation, causing enlargement and tenderness of the liver. The spleen and kidneys were also congested to a less extent; but the backward pressure in the line of the circulation had gone beyond this, and was affecting the systemic veins, demonstrated by the above mentioned cedema. The urine was very greatly diminished in quantity, sp. or. 1026, and contained a very large amount of albumen. The diagnosis was mitral regurgitation, with failure in compensation, \&c. The medicinal treatment was the exhibition of the tinctures of strophanthus and nux vomica. Compensation was soon obtained under this treatment, the pulse gialually becoming regular and strong. The backward pressure was relieved, and the congestion of the internal organs and odema entirely disappeared. When she left off taking the strophanthus there was no dyspnœa or palpitation, no albumen in the urine, and she was well, with the exception that her mitral murmur was still audible.

CASE 3.-E. $\mathrm{O}-$, aged forty-seven, was complaining of a violent pain below the left nipple, together with dyspncea and palpitation. He came to me for the first time on Jan. 2lst, complaining of the pain only, which $I$ then thought was of a neurosal character, and treated him with sedatives, which afforded him relief for some time. In March he came under the observation of my locum tenens, seeking advice for a return of the same pain, and he was prescribed something similar to what I had previously given him. He resumed work after a fortnight's rest. But he was not destined for a prolonged release of his symptom, and at the end of April I was again consulted, when the other signs above mentioned had become manifested. His height was $5 \mathrm{ft} .4 \mathrm{in}$, and his weight $10 \mathrm{st}$. $11 \mathrm{lb}$. He was well developed and muscular. There was commencing cyanosis of the alre nasi, lips, and helix of the ears Temperament neurotic; tongue slightly coated at its back part. There had been developed an undulatory systolic movement in the upper part of the epigastrium. 'The apex beat was altered in position, and was thrown into the sixth intercostal space four inches and a half from the middle line of the body and outside the nipple line. Percussion showed that the cardiac dulness was increased downwards, and a little to the left. A systolic mitral murmur, which Thad not detected before, was quite audible. It was propagated with the greatest intensity towards the axilla and back, where it became lost. Pulse rapid, weak, and very compressible. Both his lungs were now congested, especially at the bases posteriorly Urine nornal. Diagnosis : Mitral reflux, with commencing failure in compensation. Consequently, I deemed it advisable to try strophanthus, and an pleased to say the result was most gratifying. The patient resumed work on May 29th, and still continues to follow his emplovment

CASE 4.--Mrs. F-, aged forty-seven, on May 17th complained of being very weak and faint, with some diarrhoa. On examination, I found that she was a tall woman, 
well developed, but with her muscles in a very flabby condition. She was lying flat on her back in bed in an arynamic state, with the face pale and expressionless. Her diathesis was not well marked. Temperature $101 \cdot 4^{\circ}$. Tongue a little coated, very flabby, and with the fungiform papillir abnormally raised. Appetite bad, with thirst, and the odour of her breath disagreeable. There was tenderness on pressure in the epigastrium, but in every other respect the abdomen was normal. 'There was no visible apex beat, and the cardiac impulse was almost imperceptible to palpation. The first cardiac sound was inaudible, with some accentuation of the second heart sound in the mitral area. There were no murmurs present, and the action of the cardiac muscle was extremely weak. Pulse 110, rhythm regular, and in character very small and weak, with deficient filling of the arteries. I gave her strophanthus and nux vomica, together with astringent powders for her diarrhoca, and her case ended favourably on June $3 \mathrm{rd}$, when she was crossed off my list in an excellent state of health.

CASE 5.-MIrs. B-, ared fifty-seven years, a chronic invalid, who complained of a variety of variable symptoms, but principally of dyspncea and weakness. I visited her for the first time on Noy. 13th last, when she presented the following condition. A short, stout woman, rather pale in the face, with a melancholic expression. Lips pale, of a bluish colour, and the teeth mostly all decayed. The tongue was coated down the centre with a yellowish-brown fur, heing clean at each side of this band. There was anorexia, some thirst, with a sense of flatulent distension, and at times nausea and even vomiting. The liver appeared to be a little roughened on palpation, contracted slightly, and was tender to pressure. No impulse could be felt in the procordia, and the quality of the heart sounds was very weak on auscultation in the various areas. The pulse was normal in frequency, but remarkably weak and compressible. The other systems were normal. This patient had been greatly addicted to drink for years, and was found to be suffering from chronic dipsomania, with cirrhosis of her liver. Owing to the extremely weakened condition of her circulatory system, I determined to try her with the following mixture: Tincture of strophanthus, $1 \mathrm{dr}$. tincture of nux vomica, $1 \frac{1}{2} \mathrm{dr}$. ; tincture of capsicum, $1 \mathrm{dr}$. ; decoction of cinchona to $8 \mathrm{oz}$.; two tablespoonfuls to be taken thrice daily. She received considerable benefit from taking this mixture, especially so far as her circulatory system was concerned. Her heart improved so much as to be felt on palpation, and her craving for alcoholic drinks was not by any means so great. I think that possibly her desire for so much alcohol was in a great measure due to the weakened state of her circulation, with an improper flling of her cerebral arteries causing anæemia of the brain and in order to arouse this flagging circulation she drank alcohol freely.

CASE 6.-H. S—, aged fifty-nine, complained of being short of breath on the least exertion, or in stooping to lace his boots, or even to pick anything from the floor. $\mathrm{He}$ also felt a pain in the region of the heart. He had suffered from winter bronchitis for the last four years. He was a short, excessively stout person, with a pale face and gouty diathesis. It required very careful palpation to detect any impulse of the heart, as it was so very weak in its action, but natural in position. In quality the cardiac sounds were very weak at the different areas, especially the first sound in the mitral area. Pulse 78, regular, but weak, soft, and very compressible. The arteries were not degenerated. Diagnosis; Adipose infiltration upon and between the cardiac muscular fibres, causing this heart failure. The tincture of strophanthus and nux vomica, in five-minim doses of each three times a day, afforded him with very great relief. His lips have now become red, being pale and blue before. The face has also assumed a ruddy appearance. The cardiac muscle acts strongly, and the apex beat can be distinctly felt on palpation. The pulse is now 70 , very much stronger in character, and the tone is excellent.

CASE 7.-G.S-, aged thirty-four years, came under my care in September, 1887, suffering from enteric fever, having had a very severe attack of this fever, lasting for fifty-three days, and with the intervention of several conplications, the principal of them being pneumonia, hremorthage from the bowels, and thrombosis of the veins of the right leg. On Oct. 27th his circulation became very weak, and he was in imminent danger of death from cardiac failure. The state of his circulatory system was then as follows: Faintness and dyspncea, with a marked tendency to syncope The apex beat was impercentilsle to palpation. In the mitral area both the heart sounds were inaudible; but as the cup of the stethoscope was advanced towards the base of the heart the second sound gradually became audible, and it was distinctly so in the aortic area. Pulse 79 , intermittent in rhythm, and remarkably feeble. The arteries were imperfectly filled with blood. The severity of the enteric fever had undoubtedly left this patient with a considerable amount of fatty degeneration of the muscular fibres of the heart. He was ordered a mixture containing a minim and a half of the tincture of strophanthus, combined with nux vomica, every half-hour. As he improved, the medicine was given less often, being ordered every hour, and afterwards every two and four hours. In the seventh mixture the nux vomica was left out entirely, and it was prescribed to be taken three times a day. This medicine evidently saved his life, and he resumed his usual employment last February. He continues to work, and now feels as strong as he ever did.

Remarks. - The most interesting features connected with the first, second, and the sixth cases are derived from the fact of the patients having taken large quantities of digitalis from time to time under the treatment of different medical gentlemen, and this without any very great improvement in any of them. With both of the first two patients I immediately supplanted digitalis by strophanthus; consequently I was in a position to weigh accurately from a clinical aspect the effect of these two drugs in mitral disease. I believe that I can conscientionsly assert that our new remedy is vastly superior to the older one (digitalis), especially in mitral complaints and cardiac failures. It thoroughly established compensation, and caused extremely irregular and also intermittent pulses to become perfectly regular, in cases where digitalis had previously failed. It appears to act as a cardiac tonic by stimulating the cardiac muscle into a greater state of contraction, and also by prolonging this state of systole. Acting thus, it raises the blood pressure within the arteries in the various organs, as manifested by the diuresis produced in these cases; and in the fifth case by raising the tone of the cerebral circulation. It also seems to slow the rhythm of the heart, and to steady its diastole, giving it more time to nourish itself through the coronary arteries. In my opinion, when a patient is properly under theinfluence of strophanthus, it prevents an already dilated heart from assuming the same amount of dilatation after each systole as there previously existed, and this depends on the power this drug possesses in maintaining the cardiac muscular fibres in a state of contraction. I have never found strophanthus to cause the heart to beat irregularly, or to make the pulse more rapid in action, even when given in very large and continued doses, as is repeatedly observed with large doses of digitalis; neither is it cumulative in its action. The addition of nux vomica seems to accelerate and aid the therapeutical effects of strophanthus hispidus.

Anglesey.

\section{CASE OF EXCAVATED MALIGNANT TUMOUR OF THE LUNG.}

BY GRAHAM STEELL, M.D.,

ASSISTANT PHYSICIAN TO THE MAYCHESTER ROYAL INFIRMARY.

W. L_- aged forty-five, a tramcar guard, was admitted into the Manchester Infirmary on Feb. 27th, 1888. He had been in good health till May, 1887, when he suffered from an attack of hæmoptysis, for which he was treated in the infirmary. From this time his health gradually deteriorated, although cough and expectoration did not become features of his illness till the beginning of winter. He began to attend the out-patient room of the infirmary in August, when no physical sign of pulmonary disease could be detected; notwithstanding this fact and the absence of a tubercular family history, the case was regarded as one of phthisis. He attended more or less regularly during the winter, but his chest was not again examined for some time. At one of his visits he complained of a good deal of pain in his chest, and on examining it I was surprised to find the following physical signs: absolute dulness of the upper portion of the right lung, accompanied by great sense of resistance on percussion, and absence of breath sounds over 